\section{Neuroimaging of Movement Disorders}

\author{
F.B. Nahab and N. Hattori, Eds.
}

New York, NY: Springer, 2013, 290 pages, $\$ 189$

This book is a good overview of the key role that noninvasive neuroimaging plays in the diagnosis of movement disorders, which are increasingly being seen because of longer life spans. Chapter 1 provides the basics on the use of MR imaging and emission tomography such as SPECT and PET/CT for diagnosing and guiding therapies and discusses future neuroimaging modalities such as PET/MR imaging.

The second chapter, on morphometric analysis, describes promising results for MR imaging using individual structural data. Technical developments in computational neuroanatomy, especially regarding white matter applications, appear promising in the diagnosis of movement disorders. Chapter 3 describes preliminary encouraging success with diffusion MR imaging in Parkinson disease (PD). PET/ SPECT research into neural substrates involved in the pathophysiology of gait disorders, voluntary movements such as walking, and gait activation studies is covered in Chapter 4. This chapter also discusses how the combination of imaging with neurorehabilitative techniques and braincomputer interfaces can optimize outcomes by increasing our understanding of responses to therapies. In addition, the chapter covers the way in which improvements in dynamic SPECT/CT and PET can lead to a better understanding of the pathophysiology of gait disorders, including the neural substrates involved in voluntary movements.

The application of transcranial ultrasonography using high-end ultrasound devices for the workup of PD, atypical $\mathrm{PD}$, normal-pressure hydrocephalus, essential tremor, major depression, Wilson disease, restless leg syndrome, and dystonia is discussed in Chapter 5. Pathophysiologic changes underlying substantia nigra hyperechogenicity, iron content, and microglial activation are also covered. Chapter 6 discusses the clinical applications of near-infrared spectroscopy in movement disorders, including cortical activation during routine activities or treatment-augmented rehabilitation.

Chapter 7 is an overview of structural MR imaging, including clinical and research applications in idiopathic PD. Table 7, which shows MR imaging findings in various neurodegenerative diseases, is helpful. Dopamine imaging using PET and SPECT, covered in Chapter 8 , is based on cell

COPYRIGHT $\odot 2014$ by the Society of Nuclear Medicine and Molecular Imaging, Inc. loss in the substantia nigra pars compacta, with corresponding loss of striatal terminals. The chapter discusses the usefulness of this technique for differentiating idiopathic PD from other nonparkinsonian tremors, which are seen on scans as progressive reduced activity in the caudate putamen, followed by the rostral putamen, and lastly the caudate nucleus. Chapter 9 describes noninvasive functional MR imaging to demonstrate regional brain function and also discusses ongoing research to improve early diagnosis and differential diagnosis of idiopathic PD from other parkinsonism diseases or to evaluate disease progression and identify novel targets for future therapies.

Chapter 10 covers neuromelanin-sensitive imaging in idiopathic PD using specific MR imaging techniques to directly assess pathologic and functional changes in neuromelanin-containing neurons of the substantia nigra pars compacta and the locus ceruleus. Imaging techniques, normal findings, and clinical applications of neuromelaninsensitive MR imaging, including its future potential in preclinical and early diagnosis, differential diagnosis, and evaluation of drug responses in idiopathic PD, are discussed. Neuroimaging of dystonias using T1-weighted MR imaging and morphometric techniques to evaluate structural changes in gray matter; diffusion tensor imaging to identify white matter changes; PET imaging to assess changes in blood flow, function, and metabolism; and functional MR imaging to analyze brain function, connectivity, and network interactions are described in Chapter 11. The usefulness of ${ }^{18} \mathrm{~F}$-dihydroxyphenylalanine PET imaging, dopamine transporter SPECT imaging, and functional MR imaging to differentiate idiopathic PD from essential tremor is discussed. The high specificity of transcranial ultrasonography to reliably differentiate essential tremor from idiopathic PD and other imaging modalities such as MR spectroscopy, transcranial ultrasonography, and magnetoencephalography are covered in Chapter 12. Magnetoencephalography mapping of magnetic fields of naturally occurring brain activity by using special superconducting interference devices has identified the inferior olive, cerebellar nuclei, and thalamus as playing key roles in the tremor network.

Chapter 13, on neuroimaging in Huntington disease and other choreas, discusses the potential of CT, MR imaging, diffusion tensor imaging, functional MR imaging, PET, SPECT and ultrasound to assess efficacy and therapeutic interventions in choreas. Chapter 14, on neuroimaging in ataxia, discusses the use of MR imaging, functional MR imaging, and MR spectroscopy to demonstrate regions of involvement and other characteristic findings useful in differentiating the various ataxias. Dopamine transporter SPECT may show reduced striatal binding similar to idiopathic PD in 
some ataxias, whereas ${ }^{18}$ F-FDG PET may show subclinical decreased uptake in the cerebellar hemispheres; brain stem; and occipital, parietal, and temporal cortices in certain spinocerebellar ataxias. Technical advances in imaging hardware and image analysis can help us better understand the various ataxias. Chapter 15 is on MR imaging of less frequently seen gait disorders such as mesencephalic gait failure, CreutzfeldtJakob disease, freezing gait, acute lacunar stroke, posterior long ligament calcification, and PET imaging in thalamic astasia and frontotemporal dementia. PET and functional MR imaging research into brain metabolism and receptor plasticity may improve our understanding of the brain mechanisms involved in repairing defective networks affecting gait and posture and help in designing novel treatment approaches for gait disorders. Chapter 16 describes CT and transcranial ultrasonography findings in basal ganglia calcifications and describes the widespread hypoperfusion seen in subcortical and cortical regions on functional hexamethylpropyleneamine oxime imaging of bilateral striopallidodentate calcinosis patients.

This handy manual is a good introduction and clinical review of movement disorders supplemented with clinically relevant images showcasing important findings in specific movement disorders and ongoing relevant research in the evolving field. The list of abbreviations at the front of the book makes it easy to look up unfamiliar names. I highly recommend the book as a valuable addition to one's personal library and to departmental or hospital medical libraries.

Usha Joseph

University of Texas Medical School at Houston 6431 Fannin, MSB 2.130 B

Houston, TX 77030

E-mail: usha.a.joseph@uth.tmc.edu

Published online Jun. 16, 2014.

DOI: $10.2967 /$ jnumed.114.143925 